\title{
78 Lehrpraxis in der Lehrerinnen- und Lehrerbildung
}

\author{
Petra Herzmann und Matthias Proske
}

\begin{abstract}
Vorlesungen und Seminare als Normalform der Lehrpraxis der Lehrerinnen- und Lehrerbildung geraten selten in den Blick von Forschung. Werden diese untersucht, lassen sich zwei Erkenntnisinteressen unterscheiden: Zum einen die Frage nach den Wirkungen von Studium und Lehre, zum anderen die Analyse des operativen Vollzugs der Lehrpraxis. Damit verbundene Forschungszugänge weisen jedoch noch deutliche Leerstellen auf.
\end{abstract}

\section{Gegenstand und Definition}

Unter Lehrpraxis in der Lehrerinnen- und Lehrerbildung können alle Formate und darin eingelagerte Praxen verstanden werden, in denen sich Lehrerinnen- und Lehrerbildung als institutionalisierte, organisierte und intentionale Praxis operativ, d.h. in der Kommunikation zwischen Lehrpersonal und Studierenden vollzieht. In der universitären ersten Phase stellen in Präsenzform organisierte Vorlesungen und Seminare - wie auch in den meisten anderen Studiengängen die zentralen interaktionsbasierten Formate dieser Praxis dar. Erweiterungen dieser Normalform sind in drei Hinsichten zu beobachten: Erstens hat im Kontext des medialen Wandels digitale Kommunikation Eingang in die Lehrpraxis gefunden, d.h. auf universitären Lernplattformen finden sich z. B. Aufgaben, Fallmaterial, Erklärvideos oder Vorlesungen als Ergänzung oder Ersatz zur Präsenzkommunikation in Hörsälen und Seminarräumen. Zweitens ergänzen in einigen Fächern - als Ausdruck spezifischer Fach- und Wissenskulturen - Formate wie Übungen, Laborpraktika oder Exkursionen die Normalform. Und schließlich finden sich drittens im Kontext des Studienelements ,Praxisphase' meist Veranstaltungen, die deren Vorbereitung, Begleitung und Nachbereitung dienen. Ausgangspunkt dieses Kapitels ist der augenfällige Befund, dass sein Gegenstand, die Lehrpraxis in Hochschulen, im Kontext der Lehrerinnen- und Lehrerbildung einerseits in sehr spezifischer Weise in den Blick gerät und andererseits gleichzeitig nahezu unsichtbar bleibt.

Die Unsichtbarkeit der Lehrpraxis im Diskurs der Lehrerinnen- und Lehrerbildung kann daran festgemacht werden, dass datenbasierte Bestandsaufnahmen zur Normalform dieser Lehrpraxis weitgehend fehlen. Auch deshalb muss in der Definition dessen, was mit Lehrpraxis der Lehrerinnen- und Lehrerbildung gemeint ist, auf alle quantifizierenden Angaben zur Verteilung der Lehrformate verzichtet werden. Prüft man einschlägige Handbücher, Lexika oder Einführungen, die sich mit Fragen der Lehrerinnen- und Lehrerbildung (vgl. z. B. Blömeke, Reinhold, Tulodziecki \& Wildt, 2004), der Hochschullehre (vgl. z. B. Fry, Ketteridge \& Marshall, 2009) oder der Hochschulforschung (vgl. z. B. Wilkesmann, 2019) beschäftigen, ist deutlich zu erkennen, dass es keine belastbaren Daten zu Lehrformaten und deren Verteilung oder zu Lehrpraxen gibt. Dies scheint aber keine Besonderheit der Lehrerinnen- und Lehrerbildung zu sein; offensichtlich ist die alltägliche Lehrpraxis der an Universitäten und Hochschulen institutionalisier- 
ten Fächer kaum Gegenstand der Hochschulforschung. Nicht nur die Lehrerinnen- und Lehrerbildung scheint nicht zu wissen bzw. wissen zu wollen, in welchen Formaten sie mit welcher Häufigkeit lehrt und welche Praxen mit diesen Formaten der Lehre einhergehen.

Der Befund gewinnt an Schärfe, wenn man ihn in Beziehung setzt zur konträren Bedeutung hochschuldidaktischer Diskurse zur Verbesserung und Professionalisierung der universitären Lehre. Der hochschuldidaktische Blick mit seinen diskursiv verschränkten Leitbegriffen Kritik, Reform, Konzeptentwicklung, Methode und Evaluation scheint geradezu die erkenntnisleitende Prämisse jeglicher Perspektivierung von Lehrpraxis in der Hochschulforschung zu sein (vgl. Huber, 1999). Unter dem Vorzeichen von Governance konnte sich diese Perspektive mit forcierten Steuerungsmaßnahmen zur „Gestaltung und Qualitätsentwicklung“ der universitären Lehrpraxis verknüpfen, die „seit geraumer Zeit ein zentrales Thema für Hochschulen und Universitäten“ sind (Brahm, Jenert \& Euler, 2016, S. 11). Als forschungspolitische(r) Ausdruck bzw. Unterstützung einer solchen Steuerung universitärer Lehre kann etwa das 2008 aufgelegte Programm des Bundesministeriums für Bildung und Forschung (BMBF) „Hochschulforschung als Beitrag zur Professionalisierung der Hochschullehre" verstanden werden (vgl. Jahnke \& Wildt, 2011, S. 11-15).

Ohne vorschnell die irritierende Kluft zwischen fehlenden Bestandsaufnahmen und bildungspolitisch forcierten Steuerungsmaßnahmen erklären zu wollen, ist doch auf zwei systematische Merkmale der lehrbezogenen Hochschulforschung, auch bezogen auf die Lehrerinnen- und Lehrerbildung hinzuweisen: Erstens bildet die Untersuchung der Bedingungsfaktoren von Hochschullehre gegenüber programmatisch-konzeptuellen Optimierungsanstrengungen weiterhin eine Leerstelle in der Hochschulforschung (vgl. Houben, 2013). Zweitens scheint gerade die Normalpraxis der Lehre als Forschungsgegenstand deshalb so unattraktiv, weil Konzeptentwicklung und die Beforschung dieser Konzepte in einer Hand liegen (vgl. Herzmann, Kunze, Proske \& Rabenstein, 2019, S. 7-9). Diese nicht selten sogar ad personam zurechenbare Verbindung von Forschung und Entwicklung zeigt sich nicht zuletzt an der Förderlinie Qualitätsoffensive Lehrerbildung (QLB), ebenfalls des BMBF, in der neben Projekten zur Strukturentwicklung vor allem Projekte gefördert werden, in denen innovative Lehrkonzepte entwickelt, erprobt, evaluiert und damit auf einer mehr oder weniger breiten Datenbasis - evidenzbasiert weiterentwickelt werden (sollen) (Gehrmann, 2018). Die Normalpraxis von Lehre in der Lehrerinnen- und Lehrerbildung scheint in einer solchen Konstellation für die Forschenden tendenziell ebenso unattraktiv wie für die Forschungsförderorganisationen.

\section{Forschungsgegenstand}

Dass datenbasierte Bestandsaufnahmen fehlen und hochschuldidaktisch-konzeptuelle Perspektivierungen in der Beforschung der Lehrpraxis der Lehrerinnen- und Lehrerbildung dominieren, heißt jedoch nicht, dass es keine analytische Beforschung von Studium und Lehre gibt. Zwei in ihrem Gegenstandsverständnis differente Forschungsstränge lassen sich unterscheiden:

1) Einem kompetenztheoretischen Zugang ( $\rightarrow$ Kapitel 19) lassen sich Untersuchungen zuordnen, die sich für die Erträge der Lehrerinnen- und Lehrerbildung interessieren und dabei nach dem - auch vermittelt durch die Bedingungsvariable universitärer Lerngelegenheiten - erworbenen fachlichen, fachdidaktischen und pädagogischen Wissen der Studierenden fragen. Fluchtpunkt ist die Frage nach der Wirksamkeit der Lehrerinnen- und Lehrerbildung (vgl. Blömeke, 2004; Hascher, 2014), wobei Wirksamkeit verstanden wird als Verhältnis zwischen Merkmalen der Ausbildung auf der Systemebene - hier Lehrorganisation und ggf. Lehrpraxis - und dem Output in Gestalt messbarer Kompetenzentwicklung auf der Individualebene 
(vgl. König \& Klemenz, 2015). Auch die meisten Evaluations- bzw. Entwicklungsstudien in der QLB können diesem Strang zugeordnet werden. Deutliche Schnittmengen mit neueren, sich an der Lehr-Lern-Forschung orientierenden hochschuldidaktischen Untersuchungen im Kontext der Hochschulforschung sind ebenfalls zu erkennen (vgl. Winteler \& Förster, 2007; Merkt, 2014).

2) Einem praxistheoretischen Forschungsstrang ( $\rightarrow$ Kapitel 22) lassen sich Untersuchungen zuordnen, die zum einen nach dem operativen Vollzug der Lehrpraxis fragen und analysieren, was universitäre Interaktionspraxis in ihrem alltäglichen Tun konstituiert. Zum anderen zielt praxistheoretische Forschung ebenfalls auf Erträge universitärer Lehrpraxis für Professionalisierungsprozesse der Studierenden (im Überblick Herzmann et al., 2019) im Sinne der „Sozialisation in die institutionelle und kommunikative Kultur der akademischen Gemeinschaft" (Tyagunova, 2019, S. 2).

\subsection{Forschung zur Wirksamkeit von Studium und Lehre}

In ihrem Überblick zur Wirksamkeit und Qualität der Hochschullehre weisen Metz-Göckel, Kamphans und Scholkmann (2012) die Untersuchung der Kompetenzentwicklung der Studierenden mit Beginn der 2000er Jahre als zentralen empirischen Zugang zur universitären Lehrqualität aus. Von dieser „personenzentrierte[n] Forschung“ (S. 219) grenzen sie zwei weitere Forschungsrichtungen ab: Zum einen Forschungen zur Wirksamkeit unterschiedlicher Lehrformate, wobei traditionelle Lehrformate häufig mit konstruktivistischen Formaten (wie z.B. dem Forschenden Lernen) verglichen werden. Zum anderen Interventionsforschungen, die die Wirksamkeit einzelner seminardidaktischer Maßnahmen untersuchen und z. B. das gezielte Fragenstellen oder die Wahlfreiheit bei der Bearbeitung von Aufgaben in einer Vorlesung mit dem Lernerfolg der Studierenden in einen Zusammenhang stellen (z. B. Kordts-Freudinger, Klingsieck \& Seifried, 2018). Für beide Forschungsrichtungen wird die Befundlage als äußerst heterogen eingeschätzt und mit Blick auf die Komplexität der universitären Lehre wird keine Eindeutigkeit hinsichtlich der grundsätzlichen Überlegenheit eines Formats (instruktionsorientiert oder konstruktivistisch) festgestellt (Metz-Göckel et al., 2012). Mittels einer Videoanalyse von Hochschulseminaren konnten Seidel und Hoppert (2011) allerdings zeigen, dass deren Gestaltung durch hohe Redeanteile der Lehrenden und lange Instruktionsphasen geprägt ist und sich diesbezüglich keine signifikanten Unterschiede zwischen den untersuchten Fakultäten feststellen lassen.

In der Forschung zur Kompetenzentwicklung der Studierenden stellen die Ausbildungsstandards der Kultusministerkonferenz (KMK, 2004) den Maßstab der zu erwerbenden Fähigkeiten in der universitären Lehrerinnen- und Lehrerbildung dar. Ziel ist es zu prüfen, inwieweit diese Standards erreicht werden und ggf. welche Effekte auf einzelne Studienteile (z. B. Praktika) zurückzuführen sind (vgl. Kuhn, Zlatkin-Troitschanskaia, Pant \& Hannover, 2016). Ohne Befunde hier berichten zu können, zeigt sich, dass die Modellierung und Messung kognitiver Dispositionen und fachspezifischen Wissens im Rahmen von Leistungsstudien gelingen. Dennoch wird wiederholt auf unzulässige Kausalbeziehungen von Lehrpraxis und Wissenserwerb hingewiesen, in dessen Konsequenz eine gewisse Ernüchterung steht: „Im Grunde wissen wir wenig darüber, welche Wirkungen die Lehrveranstaltungen in ihren unterschiedlichen Formaten hervorbringen, was Studierende in den konkreten Lehrveranstaltungen tatsächlich lernen und wie viel also auf die Lehrveranstaltung selbst zurückzuführen ist" (Metz-Göckel et al., 2012, S. 218). Zu konstatieren ist außerdem, dass aufgrund der Ausrichtung der Kompetenzforschung an psychologischen Messverfahren organisationale Perspektiven auf institutionelle Vermittlungsstrukturen sowie auf die formale Organisation Hochschule im Kontext von Wirksamkeitsstudien selten verfolgt werden (Houben 2013; Merkt, 2014). 


\subsection{Forschung zum Vollzug der Lehrpraxis}

Praxistheoretische Arbeiten zur Lehrpraxis richten ihren Blick auf das interaktive Geschehen in der Hochschullehre und intendieren, häufig aus einer ethnomethodologischen Perspektive, „deren Spezifik in Details der Interaktionsarbeit nachzuvollziehen und zu beschreiben“" (Tyagunova, 2019, S. 2). Untersucht wird etwa, welche Formen der Teilnahme von Studierenden an einem universitären Seminar praktiziert werden und welchen Einfluss Studierende dadurch auf die Konstitution der seminaristischen Ordnung nehmen (Tyagunova, 2017). Mit der Kohärenz von (kommunikativen) Beiträgen in einem Seminar beschäftigt sich die Untersuchung von König (2019), die ein deutliches Spannungsverhältnis nachweist zwischen den diskursiven Ansprüchen seminaristischer Praxis und dem studentischen Scheitern, inhaltliche (Gesprächs-)Anschlüsse systematisch herzustellen. Explizit bezogen auf die seminaristische Kommunikation von Lehramtsstudierenden ist die Untersuchung von Wenzl (2019), die in erziehungswissenschaftlichen Lehrveranstaltungen studentische Orientierungen an geselligem Austausch, aber nur lose $\mathrm{Zu}$ sammenhänge mit den im Seminar behandelten Themen rekonstruiert. Die Selbstverständlichkeit, mit der Lehramtsstudierende sich als Mitglieder einer Gruppe ,Gleichgesinnter ${ }^{\text {' }}$ - nicht etwa vornehmlich als Teilnehmende eines fachwissenschaftlichen Diskurses - positionieren, interpretiert Wenzl (2019) als „Ausdruck eines Beheimatungsproblems“ (S. 25) der Lehramtsstudierenden an der Universität. Für Kommunikationsprozesse in Seminaren der universitären Lehrerinnen- und Lehrerbildung interessieren sich auch die Studien von Artmann, Herzmann, Hoffmann und Proske (2017) sowie Bräuer, Kunze und Rabenstein (2018), die die Herstellung der Gültigkeit von Deutungen im studentischen Sprechen über Unterricht untersuchen. Dabei werden im ersten Fall in einer funktionalen Herangehensweise Bezugsprobleme wie die Kommunikation von Aufgabenstellungen, die Versprachlichung von pädagogischem Wissen und die Herstellung von Gültigkeiten (Artmann et al., 2017) analysiert, die von Studierenden und Lehrenden bearbeitet werden müssen, um die öffentlich organisierte Seminarinteraktion und deren Fortsetzung zu ermöglichen. In der Studie von Bräuer et al. (2018) werden demgegenüber sprachanalytische Muster der Bearbeitung von Geltungsfragen in Gruppenarbeitsprozessen untersucht und auch hier zeigt sich u. a. - analog zu den Befunden von Wenzl (2019) - ein InGeltung-Setzen von Deutungen qua subjektiver Zustimmung aufgrund eigener Erfahrungen.

Für die bisher vorliegenden Studien des praxistheoretischen Forschungsprogramms (weiterführend Herzmann et al., 2019) fällt zweierlei auf: Erstens machen die Studien einen Detailreichtum lokaler und kommunikativer Praktiken in der Lehre sichtbar, die systematische Einordnung der Befunde in den institutionellen Kontext universitärer Lehrerinnen- und Lehrerbildung steht aber noch aus. Zweitens arbeiten die Untersuchungen vor allem das Nichterfüllen diskursiver Ansprüche in Seminaren heraus. Erwartungen an Professionalisierung von Lehramtsstudierenden werden in augenfälliger Krisenhaftigkeit sichtbar gemacht, so dass die Risiken studentischer Sozialisation beschrieben werden können.

\section{Diskussion}

Der Forschungsstand zum Normalfall akademischer Lehre in der Lehrerinnen- und Lehrerbildung ist sowohl mit Blick auf dessen operativen Vollzug (Herzmann et al., 2019) wie dessen Qualität (Spinath \& Seifried, 2018) dürftig. Auf Gründe dafür haben wir einleitend hingewiesen.

Bilanziert man die Erträge der vorhandenen Forschung, lässt sich für den kompetenzorientierten Ansatz vor allem ein präziserer Einblick in die zeitliche Genese und die domänenspezifischen Unterschiede innerhalb des professionellen Wissens von angehenden Lehrpersonen festhalten. Für den praxistheoretischen Ansatz wiederum ist die mikrologische Aufklärung all- 
täglicher Interaktionsroutinen und deren Bezug zur Produktion von Wissen der Lehrerinnenund Lehrerbildung insbesondere im Format des Hochschulseminars kennzeichnend.

Insofern aktuell aber eine intensivere Beforschung des Lehramtsstudiums zu beobachten ist, scheint es notwendig, bestehende Leerstellen der bisherigen Ansätze zu markieren. Gerade wenn man von einer Wirkungskette mit den Elementen intendiertes, realisiertes und angeeignetes Curriculum der Lehrerinnen- und Lehrerbildung ausgeht ( $\rightarrow$ Kapitel 94), können Untersuchungen im Spektrum der kompetenzorientierten Wirkungsforschung in Zukunft nicht darauf verzichten, die operative Realisierung und Nutzung der institutionell bereitgestellten Lerngelegenheiten in deren Differenzen und Komplexitäten systematisch deutlich stärker in ihren Studien zu berücksichtigen. Spinath und Seifried (2018) plädieren i. d. S. „dafür, mehrere Kriterien in einer Untersuchung zu berücksichtigen (sowohl dem Inhalt als auch der Erhebungsmethode nach), um dadurch ein Gesamtbild guter Hochschullehre zu definieren und untersuchen zu können" (S. 158-159). Über Einzelbefunde hinaus hätten praxistheoretische Forschungsarbeiten ihre Designs demgegenüber deutlich stärker darauf auszurichten, dass die Einordnung und Kontextuierung lokaler Lehrpraxen in ihre institutionellen Rahmungen möglich wird. Insbesondere die funktionale Analyse von Bezugsproblemen der Lehrpraxis und deren Bearbeitung durch die beteiligten Akteurinnen und Akteure und Organisationseinheiten, d. h. die systematische Verknüpfung einer struktur- und einer prozesstheoretischen Perspektive auf universitäre Lehrpraxis, scheint dringend geboten. Bisherigen Studien in beiden Zugängen fehlt zudem meist die Anbindung an eine organisationstheoretisch fundierte Governanceperspektive (vgl. Hüther \& Krücken, 2016; Wilkesmann, 2019), damit die Rahmenbedingungen der Vollzugslogiken der Lehrpraxis bestimmbar werden. Hier ist nicht nur an das komplexe Verhältnis von Wissenschafts- und Bildungspolitik im Bereich der Lehrerinnen- und Lehrerbildung, sondern auch an die vielfältigen organisatorischen Konstellationen innerhalb der Universität zwischen Verwaltung, Fakultäten und weiteren Akteurinnen und Akteuren (z. B. Zentren für Lehrerbildung) zu denken. Als institutionelle Handlungsbedingungen strukturieren sie die Lehrpraxen ebenso mit wie die Lern- und Bildungsprozesse der Studierenden.

Methodologisch blind ist die Forschung zur Lehrpraxis gegenüber ihrer doppelten Involvierung als (Hochschul-)Lehrende der Lehrerinnen- und Lehrerbildung und (Hochschul-) Forschende zur Lehrerinnen- und Lehrerbildung. Deren Implikationen werden bislang weder thematisiert noch Untersuchungsdesigns und Ergebnisse in Bezug auf mögliche Engführungen aufgrund dieser doppelten Positionierung geprüft.

\section{Empfehlungen zur vertiefenden Lektüre}

Herzmann, P., Kunze, K., Proske, M., \& Rabenstein, K. (2019). Die Praxis der Lehrer*innenbildung. Ansätze - Erträge - Perspektiven. Zeitschrift für interpretative Schulund Unterrichtsforschung, 8, 3-23.

Spinath, B., \& Seifried, E. (2018). Was brauchen wir, um solide empirische Erkenntnisse über gute Hochschullehre zu erhalten? Zeitschrift für Hochschulentwicklung, 13(1), 153-169.

Wilkesmann, U. (2019). Methoden der Hochschulforschung. Eine methodische, erkenntnis- und organisationstheoretische Einführung. Weinheim: Beltz Juventa. 


\section{Literatur}

Artmann, M., Herzmann, P., Hoffmann, M., \& Proske, M. (2017). Sprechen über Unterricht. Wissenskommunikation in kasuistischen Formaten der universitären Lehrerbildung. Lehrerbildung auf dem Prüfstand, 10(2), 216-233.

Blömeke, S. (2004). Empirische Befunde zur Wirksamkeit der Lehrerbildung. In S. Blömeke, P. Reinhold, G. Tulodziecki \& J. Wildt (Hrsg.), Handbuch Lehrerbildung (S. 59-91). Bad Heilbrunn: Klinkhardt.

Blömeke, S., Reinhold, P., Tulodziecki, G., \& Wildt, J. (Hrsg.) (2004). Handbuch Lehrerbildung. Bad Heilbrunn: Klinkhardt.

Brahm, T., Jenert, T., \& Euler, D. (Hrsg.) (2016). Pädagogische Hochschulentwicklung: Von der Programmatik zur Implementierung. Wiesbaden: Springer VS.

Bräuer, C., Kunze, K., \& Rabenstein, K. (2018). Positionierungen im Sprechen über Unterricht. In T. Leonhard, J. Kosinár \& C. Reintjes (Hrsg.), Institutionelle Praktiken und Orientierungen in der Lehrerinnen- und Lehrerbildung (S. 139-154). Bad Heilbrunn: Klinkhardt.

Fry, H., Ketteridge, S., \& Marshall, S. (Eds.) (2009). A Handbook for Teaching and Learning in Higher Education (3rd ed.). London: Routledge.

Gehrmann, A. (2018). Top-down versus Bottom-up? Die Qualitätsoffensive Lehrerbildung zwischen Pazifizierungsstrategie und kohärentem Programm. journal für lehrerInnenbildung, 18(3), 9-20.

Hascher, T. (2014). Forschung zur Wirksamkeit der Lehrerbildung. In E. Terhart, H. Bennewitz \& M. Rothland (Hrsg.), Handbuch der Forschung zum Lehrerberuf (2. Aufl., S. 542-570). Münster: Waxmann.

Herzmann, P., Kunze, K., Proske, M., \& Rabenstein, K. (2019). Die Praxis der Lehrer*innenbildung. Ansätze - Erträge Perspektiven. Zeitschrift für interpretative Schul- und Unterrichtsforschung, 8, 3-23.

Houben, D. (2013). Governance der Hochschullehre. Swiss Journal of Sociology, 39(2), 361-381.

Huber, L. (1999). An- und Aussichten der Hochschuldidaktik. Zeitschrift für Pädagogik, 45(1), $25-44$.

Hüther, O., \& Krücken, G. (2016). Hochschulen. Fragestellungen, Ergebnisse und Perspektiven der Hochschulforschung. Wiesbaden: Springer VS.

Jahnke, I., \& Wildt, J. (2011). Hochschuldidaktische Hochschulforschung - fachbezogen und fachübergreifend?! In I. Jahnke \& J. Wildt (Hrsg.), Fachübergreifende und fachbezogene Hochschuldidaktik (S. 9-18). Bielefeld: Bertelsmann.

König, H. (2019). Die Unwahrscheinlichkeit der Kohärenz. Mikrologische Untersuchungen der Struktur kommunikativer Anschlüsse im universitären Seminardiskurs. In T. Tyagunova (Hrsg.), Studentische Praxis und universitäre Interaktionskultur (S. 29-56). Wiesbaden: Springer VS.

König, J., \& Klemenz, S. (2015). Der Erwerb von pädagogischem Wissen bei angehenden Lehrkräften in unterschiedlichen Ausbildungskontexten. Zeitschrift für Erziehungswissenschaft, 18(2), 247-277.

Kordts-Freudinger, R., Klingsieck, A. B., \& Seifried, E. (2018). Beforschung einer eigenen Vorlesung: Effekte von Wahlfreiheit auf Leistung und Konfidenz. Zeitschrift für Hochschulentwicklung, 13(1), 85-104.

Kuhn, C., Zlatkin-Troitschanskaia, O., Pant, H.A., \& Hannover, B. (2016). Valide Erfassung der Kompetenzen von Studierenden in der Hochschulbildung. Zeitschrift für Erziehungswissenschaft, 19(2), 275-298.

KMK [Kultusministerkonferenz] (2004). Standards für die Lehrerbildung: Bildungswissenschaften. Bonn: KMK.

Merkt, M. (2014). Hochschuldidaktik und Hochschulforschung. Eine Annäherung über Schnittmengen. Die Hochschule: Journal für Wissenschaft und Bildung, 23(1), 92-105.

Metz-Göckel, S., Kamphans, M., \& Scholkmann, A. (2012). Hochschuldidaktische Forschung zur Lehrqualität und Lernwirksamkeit. Zeitschrift für Erziehungswissenschaft, 15(2), 213-232.

Seidel, T., \& Hoppert, A. (2011). Merkmale von Lehre an der Hochschule. Unterrichtswissenschaft, 39(2), $154-172$.

Spinath, B., \& Seifried, E. (2018). Was brauchen wir, um solide empirische Erkenntnisse über gute Hochschullehre zu erhalten? Zeitschrift für Hochschulentwicklung, 13(1), 153-169.

Tyagunova, T. (2017). Interaktionsmanagement im Seminar. Empirische Untersuchungen zu studentischen Partizipationspraktiken. Wiesbaden: Springer VS.

Tyagunova, T. (Hrsg.) (2019). Studentische Praxis und universitäre Interaktionskultur. Perspektiven einer praxeologischen Bildungsforschung. Wiesbaden: Springer VS.

Wenzl, T. (2019). Von Papas, Brüdern und Cousinen. Zum Problem lebensweltlicher Bezugnahmen in der erziehungswissenschaftlichen Lehre. In T. Tyagunova (Hrsg.), Studentische Praxis und universitäre Interaktionskultur (S. 11-27). Wiesbaden: Springer VS.

Wilkesmann, U. (2019). Methoden der Hochschulforschung. Eine methodische, erkenntnis- und organisationstheoretische Einführung. Weinheim: Beltz Juventa.

Winteler, A., \& Förster, P. (2007). Wer sagt, was gute Lehre ist? Evidenzbasiertes Lehren und Lernen. Das Hochschulwesen, 55(4), 102-109. 